\title{
Geburtshilflich-gynäkologische Gesellschaft in Wien
}

\section{Sitzung vom 14. Mai 1935}

Kahr: a) Fall von morbus Recklinghausen. 59jährige Frau. Über 4000 Knötchen an der Haut, gar keine an den Schleimhäuten. Ein Erbgang ließ sich nicht feststellen. 10 Jahre naeh der Menopause traten Blutungen auf. Uterus mit Fibro-Sarkom wird per Laparotomiam entfernt. Bald da-nach Metastasen im Schädelknochen und in der Wirbelsäule. Der histo-logische Befund macht ein Sarkom vom Nervengewebe ausgehend wahr-scheinlich.

b) Fall von Uterus-RuptuГ. Erste Schwangerschaft endete durch Forceps mit totem Kind. Zweite Schwangerschaft sectio caesarea mit normalem Verlauf. Dritte Schwangerschaft: Während der Berufsarbeit gegen Ende der Tragdauer spontane XJterusruptur. 15 Stunden nach den ersten Zeichen Laparotomie. Das Kind (3400 g) lebend in den Eihäuten außerhalb der Gebärmutter. Diese war vorne der Länge nach aufgeplatzt und lag weitaufklaffend im kleinen Becken.

Supravaginale Amputation. Mutter und Kind gesund.

In der Aussprache bemerkt Latzko, daß die Ruptur deshalb vorne stattfand, weil der Uterus nach rückwärts gegen das Rektum zu fixiert war.

2. Brings: Kraurosis vulvae bei 17jähriger Frau. Es sind erst 2 ähnliche Fälle (im 18. und 19. Lebensjahre) bekannt. Die erste Periode trat hier im 16. Lebensjahre auf und trat später nur alle 4-6 Wochen ein. Eine Umspritzung mit $1 / 2$ prozentiger Novokain-Lösung stillte die Beschwerden, brachte aber keine Heilung.

Aussprache: Boxer empfiehlt die Behandlung mit flachen Radium -trägern. Halban empfiehlt die Umspritzung mit Novocain oder Percain. Von der Voraussetzung eines Mangels an A-Vitaminen ausgehend gab er Lebertran innerlich und äußerlich. Kahr empfiehlt Desitinsalbe aufgelöst in Vigantolöl. Latzko hat den Phosphor-Lebertran in solchen Fallen schon lange angewendet. Wenn die Kraurosis nicht bald heilt, empfiehlt er gerade bei jungen Frauen die Exstirpation der Vulva. Adler sucht eine kausale Therapie und empfiehlt daher Sexualhormone. Der Gehalt des Bltites an Kalzium ist in solchen Fallen nicht geringer als sonst.

3. Frau Dora Brücke-Teleky: Ein seltener Fall von Genitalkarzinoia mit Heilung'. Man hielt die Tumoren anfangs für entzündlich. Latzko laparotomierte 1928 die damals 26jährige Frau und fand beiderseitige Ovarialzysten. Da man in deren Wand Karzinommetastasen fand ohne einen Primärtumor feststellen zu können, wurden Röntgenstrahlen angewendet. Erst 1931 traten wieder Uterusblutungen auf. Exstirpation des Uterus mit Adenokarzinom im Korpus. Röntgennachbehandlung. Patientin derzeit geheilt.

134 Sitzungsberichte aus geburtsh.-gynäkolog. Gesellschaften. 4. Milan Spitzer: Die Bedeutimg der Epitheleinschlüsse îur die weib-liche Gonorrhoe. (Erscheint ausführlich.)

Sítzung vom 21. Mai 1935. 
GebuГtshilflich-gynäkologische Гilme yon einst und jetzt. Vorführung von Filmen, die an der Universitäts-Frauenklinik in den letzten 15 Jahren aufgenommen wurden. Einleitende Worte von 0. Frankl und Erklärungen von W. Weibel. Demonstration von 2 Gruppen medizinischer Filme, deren erste aus den Jahren 1919-1920 stammt, also aus den ersten Anfängen der medizinischen Filmproduktion überhaupt. Die Aufnahmen wurden seinerzeit in der II. Frauenklinik durch die damalige staatliche Filmstelle vorgenommen. Aufnahmegerät und Beleuchtungsapparatur waren damals mächtig und schwer beweglich. Das erschwerte die Gewinnung brauchbarer Lehrfilme erheblich. Elf der damals hergestellten Filme werden vorgeführt. Sie betreffen versehiedene Geburtshilfethemen und sind an der Lebenden aufgenommen. Einige Filme zeigen geburtshilfliche Operationen, zwei sind Trickfilme und zeigen die Entwicklung des menschlichen Eies sowie den Mechanismus der verschleppten Querlage. Aueh werden interessante Anomalien des Skelettes vorgeführt. Eklampsie, Walchersche Hängelage, Technik der Untersuchung beim Prolaps. Bei den 10 neuen Filmen kam der rot und gelb empfindliche Superplanfilm zur Verwendung. Mit diesem wurden versehiedene physiologisehe und pathologische Geburtsformen aufgenommen. Zwei Filme zeigen Schädigungen von Neugeborenen. Schließlich wurden gynäkologische Operationen gefilmt, so die vaginale Hysterektomie.

Hofstutter (Wien).

Buchbesprechutg.

Alfred Labhardt, Die natürliche Rolle der Frau im Menschheitsproblem und ihre Beeinflussung durch die Kultur. Rektoratsrede. Basel 1935, Helbing und Lichtenhahn. 29 S. Preis geh. 1,10

RM.

Meisterlieh und in gewählter Sprache führt L. in der kleinen Schrift die Unterschiede, die im Kòrperbau und in der Stellung von Mann und Frau naturgegeben sind, vor Augen. Es ist nach seiner Ansicht der Streit um die höhere Wertigkeit des einen oder anderen Geschlechtes müßig. Es gibt nicht ein Minder- oder Mehr-wertig, es gibt nur ein Anders-wertig. Wir leben in einer Zeit der Unausgeglichenheit zwischen den Gesetzen der Natur und den Produkten der Kultur. Jede Entfernung der Frau von ihren natürlichen Aufgaben rächt sich unabänderlich und zwangsläufig und es kann der Mensch sich nie den höheren Gesetzen, die über ihm walten ungestraft entziehen. Nicht in dem Kampfe der Geschlechter, sondern in der gegenseitigen Ergänzung liegt der Fortschritt der Menschheit. Das sind einige Sätze aus dem frauenkundlichen Glaubensbekenntnis des Baseler Frauenklinikers, die er in seiner Rektoratsrede niedergelegt hat. Diese Worte werden bei alien, die die Stellung und Würde der Frau richtig einsehätzen und hochhalten, lauten Beifall und namentlich auch bei uns in Deutschland, wo diese Auffassung bereits zum Staatsgrundsatz erhoben ist, viel Anerkennung finden. $\quad$ L. Seitz, Frankfurt a. M. 